\title{
All-optical diode action with Thue-Morse quasiperiodic photonic crystals
}

\author{
Fabio Biancalana \\ Department of Physics and Astronomy \\ Cardiff University, Cardiff (UK)
}

October 29, 2018

\begin{abstract}
We theoretically investigate the possibility of realizing a nonlinear all-optical diode by using the unique field-localization properties (known as Anderson-Kohmoto localization) of Thue-Morse quasiperiodic 1D photonic crystals. The interplay between the intrinsic spatial asymmetry in odd-order Thue-Morse lattices and Kerr nonlinearity gives rise to sharp resonances of perfect transmission that can be used to give a polarization-insensitive, nonreciprocal propagation with a contrast close to unity for low optical intensities. Such nonlinear diode would also represent the first all-optical device which is crucially based on Anderson-like localization.
\end{abstract}

An all-optical diode (AOD) is a spatially nonreciprocal device that in the ideal case, and for a specific wavelength $\lambda$, allows the total transmission of light along the forward direction $\left(T_{\lambda}^{+}=1\right)$, and totally inhibits light propagation along the backward direction $\left(T_{\lambda}^{-}=0\right)$, yielding a unitary contrast $C=\left(T_{\lambda}^{+}-T_{\lambda}^{-}\right) /\left(T_{\lambda}^{+}+T_{\lambda}^{-}\right)=1$. AODs are widely considered to be the key components for the next generation of all-optical signal processing, in complete analogy with electronic diodes which are widely used in computers for the processing of electric signals. Replacing relatively slow electrons with photons as carriers of information would substantially increase the speed and the bandwidth of telecommunication systems, leading to a real revolution of the telecom industry.

Due to the enormous technological implications, such unidirectional propagation ('diode action') has been studied experimentally by several groups, using the most diverse schemes and experimental techniques. Photonic crystals (PCs) [1], i.e. multilayered structures which possess a definite periodicity 
of the same order of the wavelength of light, are very suitable for this kind of applications because of their ability to totally inhibit the propagation of light over well-defined frequency regions, known as photonic bandgaps (PBGs, see Refs. [1, 2]).

Especially noticeable in this direction are the early efforts of Scalora et al. [3], who in 1994 proposed the use of 1D PCs or thin films, with a gradation that makes the structure spatially asymmetric, allowing nonreciprocity due to different forward/backward nonlinear shifts of the band edges in the transmission spectrum. More recently, in 1999 Gallo and Assanto [4] proposed the use of a waveguide with an asymmetrically placed defect, which breaks the forward/backward symmetry, in presence of quadratic nonlinearities. The same authors in 2001 showed that it is possible to achieve an efficient optical diode action in periodically poled $\mathrm{LiNbO}_{3}$ waveguides, by making use of nonlinear quasi-phase matching (QPM) [5]. Other approaches involve the use of graded gain random amplifiers [6], asymmetrically apodized nonlinear microresonators [7, anisotropic liquid crystal films [8], and left-handed materials [9].

Most of the above schemes, however, suffer from some serious drawbacks which make them not suitable for commercial and large-scale applications. Relatively large physical sizes are often needed; the balance between figures of merit and optical intensities is often inadequate, and in some cases cumbersome structural designs are necessary to provide structural asymmetry.

In this paper, we discuss a novel type of AOD design based on 1D nonlinear multilayered structures arranged in a quasiperiodic or aperiodic fashion [10] (see Fig. 1 and caption). Since the first report by Schechtman et al. [12] of metallic Al-Mn alloys showing a quasiperiodic crystal structure, and the pioneering experimental works of Merlin et al. on Fibonacci and Thue-Morse GaAs-AlAs superlattices [13, a large number of studies have been devoted to 1D, 2D and 3D quasicrystals, see [10] and references therein. More specifically, we examine the diode action in Thue-Morse lattices, which exhibit isolated, sharp and high quality resonances inside the so-called pseudobandgaps, i.e. bandgap regions which are not due to translational symmetry. Thanks to the intrinsic asymmetry of odd-order Thue-Morse sequences, it is possible to achieve nonreciprocal nonlinear shifts of these 'pseudoresonances' by using small optical intensities, which combined with the small size of the structures (of the order of a few microns), would make them feasible for advanced applications and for integration on chip.

A quasiperiodic chain is defined by a recursive, deterministic generation rule $S_{0}=\{A\}, A \rightarrow f_{1}(A, B)$ and $B \rightarrow f_{2}(A, B)$, where $S_{0}$ is the initial string and $f_{1,2}(A, B)$ are two arbitrary strings of symbols $A$ and $B$. $A$ and $B$ indicate the two kinds of layer of which the structure is made of, with 


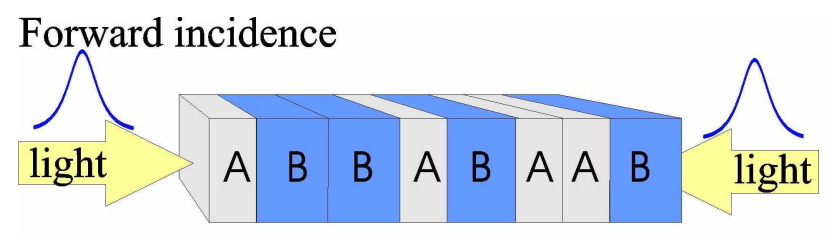

\section{Backward incidence}

Figure 1: (Color online) Geometry of the problem. Input light pulses enter the photonic crystal near normal (forward or backward) incidence. A and B indicate two dielectric materials with different refractive indices, $n_{A}$ and $n_{B}$ respectively. In this figure, the layers are arranged according to the aperiodic Thue-Morse antisymmetric sequence $\mathcal{T}_{3}$, described in the text. The nonlinear optical diode action consists in a strong asymmetry in the forward/backward transmissions.

refractive indices $n_{A}$ and $n_{B}$ respectively. Generation $S_{j}$ is a string which is constructed by applying the above recursive rule $j$ times. Several basic quasiperiodic chains have been extensively investigated in the literature, such as the Fibonacci, Cantor, Double-Period and Rudin-Shapiro sequences, see also [10] and references therein. The quasiperiodic structure that we consider here, and one of the most important in general, is the Thue-Morse sequence, which was introduced by Thue in a 1906 study [14]. It is based on the rule $\mathcal{T}_{0}=\{A\}, A \rightarrow\{A B\}, B \rightarrow\{B A\}$. The size of the string $\mathcal{T}_{j}$ for the Thue-Morse sequence is given by $2^{j}$, so that for real-life applications one usually considers $j<10$. The first Thue-Morse sequences are: $\mathcal{T}_{1}=\{A B\}$, $\mathcal{T}_{2}=\{A B B A\}, \mathcal{T}_{3}=\{A B B A B A A B\}$, etc. The ratio $L_{j}$ between number of blocks of type $A$ and number of blocks of type $B$ in $\mathcal{T}_{j}$ is always equal to unity, irrespective of the generation number $j$. It is important to note that the Thue-Morse sequence $\mathcal{T}_{j}$ is symmetric with respect to string inversion operation $\mathcal{I}$ (which is performed by reversing the order of the string elements) if $j$ is an even integer, while it is antisymmetric (i.e. it is invariant under $\mathcal{I}$ plus the substitution $A \rightarrow B$ and $B \rightarrow A$ ) if $j$ is an odd integer. In the present paper we consider only antisymmetric sequences, in that asymmetry, together with nonlinearity, is an essential requisite for the nonreciprocal behavior which is at heart of the optical diode action.

Figs. 2(a,b,c,d) show the linear transmission spectra for plane waves (as calculated with the transfer matrix method, TMM, see Ref. [2]) for the ThueMorse photonic crystals $\mathcal{T}_{3,5,7,9}$ respectively, in the wavelength range 0.5-1.1 $\mu \mathrm{m}$. Normal incidence is assumed, i.e. vanishing incidence angle $\theta=0$, so that TE- and TM-polarization spectra are degenerate. At this stage, forward and backward spectra also coincide $\left(T_{\lambda}^{+}=T_{\lambda}^{-}, \forall \lambda\right)$, since the linear transfer 


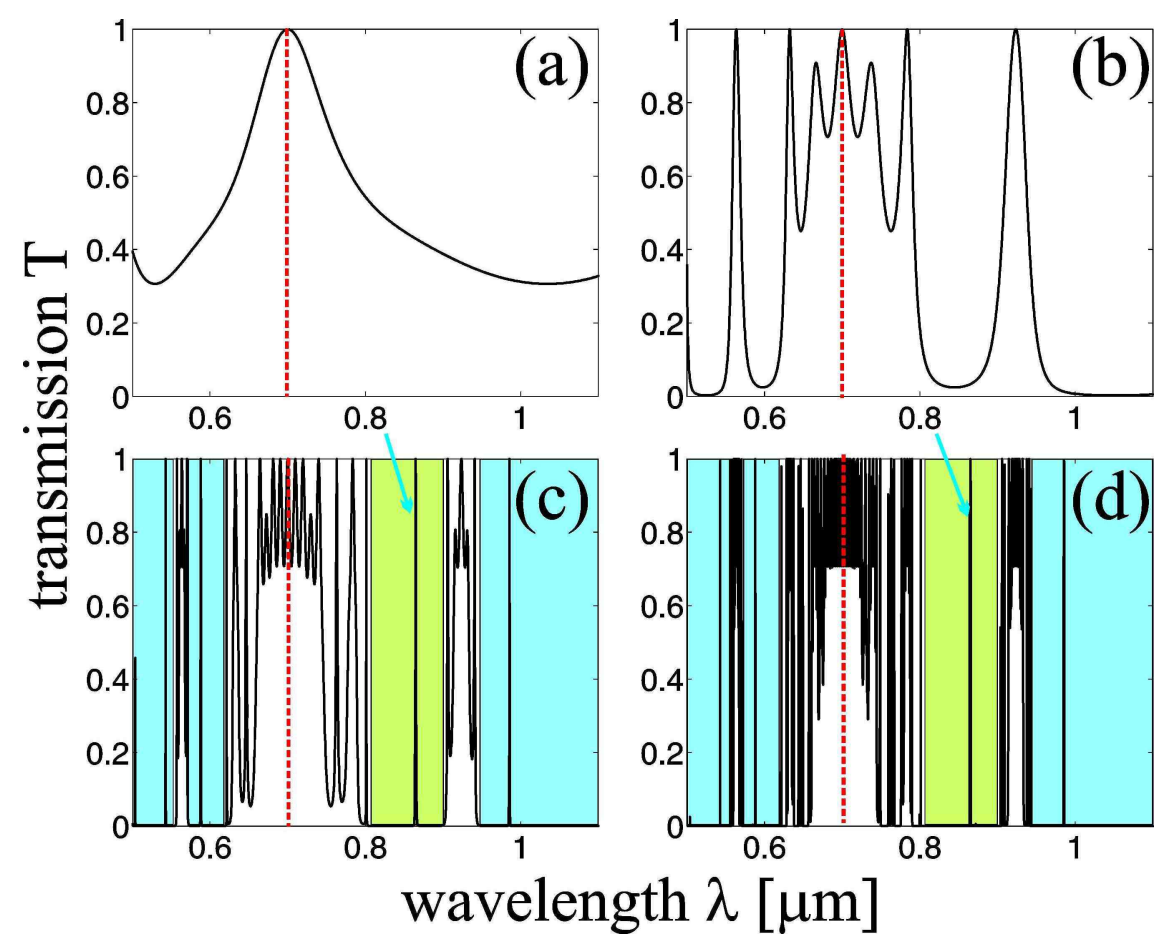

Figure 2: (Color online) Linear transmission spectra for plane waves at normal incidence $(\theta=0)$ for asymmetric odd-order Thue-Morse photonic crystals (a) $\mathcal{T}_{3}$, (b) $\mathcal{T}_{5}$, (c) $\mathcal{T}_{7}$, (d) $\mathcal{T}_{9}$. Reference wavelength $\lambda_{0}=700 \mathrm{~nm}$ is indicated with a red dashed line. The pseudobandgap and the PR of interest for this paper are indicated with a green area and a blue arrow respectively. Other large pseudobandgaps which contain other PRs are indicated with blue areas.

matrix is hermitian, and, despite the strong asymmetry of the considered lattices, no optical diode action is possible in the linear regime. Crystal parameters are: refractive indices of the layers $n_{A}=1.55$ (which corresponds to the polydiacetylene 9-BCMU organic material, see Refs. [22, 3]), $n_{B}=$ 2.3 (which corresponds to $\mathrm{TiO}_{2}$ material), $\Delta n \equiv n_{B}-n_{A}=0.75$, layer thicknesses $d_{A}=\lambda_{0} / 4 / n_{A} \simeq 112.9 \mathrm{~nm}$ and $d_{B}=\lambda_{0} / 4 / n_{B} \simeq 76.1 \mathrm{~nm}$, where $\lambda_{0} \simeq 0.7 \mu \mathrm{m}$ (indicated with a dashed red line in Fig. 2). Total physical lengths of the crystals are approximately $0.756 \mu \mathrm{m}$ for $\mathcal{T}_{3}$ [8 layers], $3.02 \mu \mathrm{m}$ for $\mathcal{T}_{5}$ [32 layers], $12.09 \mu \mathrm{m}$ for $\mathcal{T}_{7}$ [128 layers] and $48.38 \mu \mathrm{m}$ for $\mathcal{T}_{9}$ [512 layers].

Several things can be deduced from the panel of Fig. 2. First of all, it is easy to see that, when increasing the generation number $j$, each peak of transmission shows evidence of self-similarity, in the sense that it splits into other peaks, the structure of which resembles the whole spectrum. This 
fractal property of Thue-Morse spectra is a very well-known feature which is discussed in many works [11. Secondly, and most importantly, most of the peaks shown in Fig. 2 are peaks of perfect transmission (PPTs, for which $T=1$ ), regardless of the value of $j$, and they tend to accumulate around the reference frequency $\lambda_{0}$, at which the quarter wavelength condition is satisfied. Amongst the basic quasiperiodic crystals, this seems to be a unique and remarkable feature of Thue-Morse sequences. For instance, Fibonacci crystals would give, for the same choice of parameters, a small number of PPTs far from $\lambda_{0}$, and many peaks of partial transmission $(T \neq 1)$ around $\lambda_{0}$; only a symmetrization of the Fibonacci structure, of the kind described in Ref. [17, would produce PPTs, but as we have seen symmetrization is not acceptable for our purposes.

Another important feature shown in Fig. 2 is the gradual (for $j \geq 7$ ) formation of pseudobandgaps, i.e. frequency regions of zero transmission where the crystal behaves like a perfect mirror. Pseudobandgaps are perfectly analogous to conventional bandgaps, with the substantial difference that their physical origin is not due to periodicity as in Bragg reflectors. In Figs. 2(c) and2(d) one particular pseudobandgap under scrutiny is indicated with a green shaded region, while other pseudobandgaps (the ones containing other PRs) present in the spectra are indicated with blue areas. This gap contains a sharp resonance [pseudoresonance, $\mathrm{PR}$, indicated with blue arrows in Fig. 2(c) and 2(d)], analogous to a defect state, that in presence of nonlinearity can be used to obtain nonreciprocal propagation. This is located at $\lambda_{\text {res }} \simeq 0.8648 \mu \mathrm{m}$, and its existence is due to the unique field-localization features of quasiperiodic PCs, known as Anderson-Kohmoto localization [20]. Anderson-Kohmoto localization differs from proper Anderson localization in that the crystal layers are not randomly arranged. Moreover, pure Anderson localization never allows peaks of perfect transmission, and usually the number of layers necessary to achieve good transmission is very large. Therefore Anderson-Kohmoto localization maintains the good properties of periodic crystals, i.e. peaks of perfect transmission for defect states, but at the same time allows the strong asymmetry (typical of random crystals) which is necessary to design a nonlinear diode.

Resonances inside other pseudobandgaps can be used, depending on choice and availability of laser sources. It is important to recognize that such sharp defect-like resonant states are impossible to achieve by using conventional periodic PCs possessing an asymmetrically placed defect, or through apodized PCs, because this would lead only to very broad and low-quality peaks in the transmission spectra.

Quality factor (Q-factor) is a measure of the average energy in the resonant peak over the energy radiated per cycle [16]. It is defined as $Q \equiv$ 
$\lambda_{\text {res }} / \Delta \lambda$, where $\lambda_{\text {res }}$ is the central resonant wavelength and $\Delta \lambda$ is the resonance full-width at half maximum (FWHM). In Fig. 3(a) the evolution of Q-factor for the PR indicated above is shown as a function of generation number. The $\mathrm{Q}$-factors for the $\mathrm{PR}_{j}$ resonance are: $Q\left(P R_{6}\right)=262.06$, $Q\left(P R_{7}\right)=864.8, Q\left(P R_{8}\right)=1736.53$ and $Q\left(P R_{9}\right)=1235.4$. The resonance appears (together with the pseudobandgap) at $j=6$ [Fig. 33(b)], and it has a well-defined bell-shape until $j=9$ [Fig. 3](e)], at which the fractal selfreplication of the spectrum occurs. At this point the $\mathrm{PR}$ broadens due to the birth of satellite peaks. Remarkably, the central wavelength of the PR (where it exists) does not depend on $j$. For the chosen index contrast, it makes sense to talk about this PR only for $j=6,7,8$. However, we need to restrict ourselves to odd-order Thue-Morse sequences, the only ones that do not exhibit spatial symmetry. For this reason we select $\mathcal{T}_{7}$ as our representative structure, which has the best PR specifics for the selected value of $\Delta n$. In order for the pulse to clearly distinguish the resonance, its duration $\tau_{p}$ must be $\gg 2 \pi / \Delta \omega$, where $\Delta \omega$ is the resonance FWHM. In the case of $\mathrm{PR}_{7}$ we have $\Delta \omega \simeq 2.5 \mathrm{THz}$, so that a quasi-CW pulse is defined by $\tau_{p}>2.5$ psec. This, together with material relaxation time, will limit the device switching time.

Before embarking in the nonlinear analysis of the structures, it is useful to understand how small fluctuations (always present in realistic configurations) in the angle of incidence of light from perfect normal incidence $(\theta=0)$ can affect the transmission spectra. Thus it will be necessary to distinguish between the spectra of both TE- and TM-polarizations. Fig. 4 shows the $\theta$-dependence of TE- and TM-polarization spectra for the linear Thue-Morse lattice of interest, $\mathcal{T}_{7}$. Position of the $\mathrm{PR}_{7}$ peak is also indicated. For $\theta=0$, i.e. for perfect normal incidence, the two output spectra coincide, since there is no distinction between $s$ - and $p$-waves. The most important feature that can be deduced from Figs. 4 4 is the presence of minima in the peak positions in correspondence of the 'degeneracy' point $\theta=0$, a desirable feature for what concerns device applications. This leads to a considerable structural stability of the PR to small random fluctuations in $\theta$, which is also polarizationindependent to a high degree of approximation. This observation is also useful in the construction of the so-called omnidirectional PBGs, which exist for large values of $\Delta n$, see Ref. [18].

We now proceed to analyze the nonlinear behavior of the $\mathrm{PR}_{7}$ peak shown in Fig. 3(c). Figs. 5(a,b) display the different nonlinear frequency shifts of $\mathrm{PR}_{7}$ (as calculated with the nonlinear TMM, see Ref. [3]) for three different input intensity conditions $\left(I=0\right.$, black dotted lines; $I=10 \mathrm{MW} / \mathrm{cm}^{2}$, solid red lines; and $I=50 \mathrm{MW} / \mathrm{cm}^{2}$, solid blue lines), for both forward [Fig. 5(a), $T_{\lambda}^{+}$] and backward [Fig. [5(b), $T_{\lambda}^{-}$] incidence. The nonlinear Kerr 

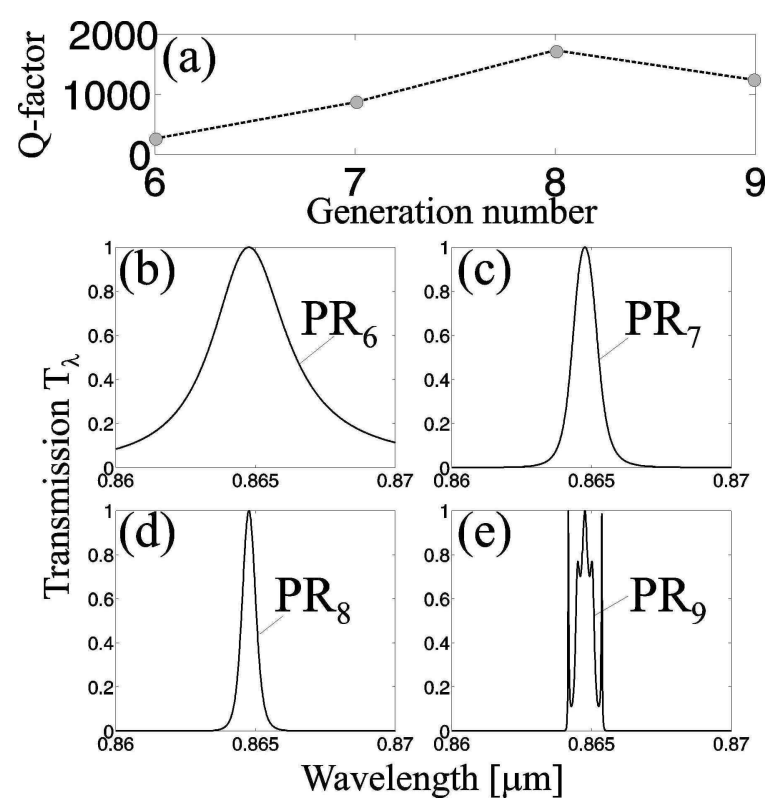

Figure 3: (Color online) (a) Q-factors of PR at $\lambda_{\text {res }}=0.8643 \mu \mathrm{m}$ as a function of generation number for Thue-Morse PCs. Crystal parameters are given in the text. (b,c,d,e) Transmission spectra of the $\lambda_{\text {res }}=0.8643 \mu \mathrm{m}$ PR for $j=6,7,8,9$ respectively. Note that for $j=9$, Fig. (e), the resonance splits and generates satellite peaks, which decreases its overall Q-factor.

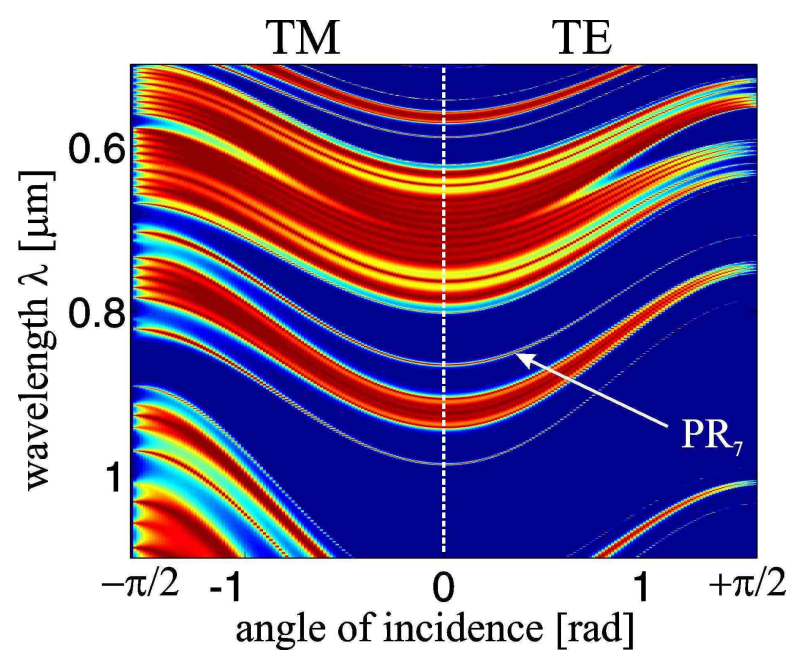

Figure 4: (Color online) $\theta$-dependence of TM- and TE-polarization spectra (left and right parts of the plot respectively) for the linear Thue-Morse quasicrystal $\mathcal{T}_{7}$. The pseudoresonance $P R_{7}$ analyzed in the text, living in one of the pseudobandgaps supported by the structure, is shown with an arrow. Large blue regions correspond to pseudobandgaps. 

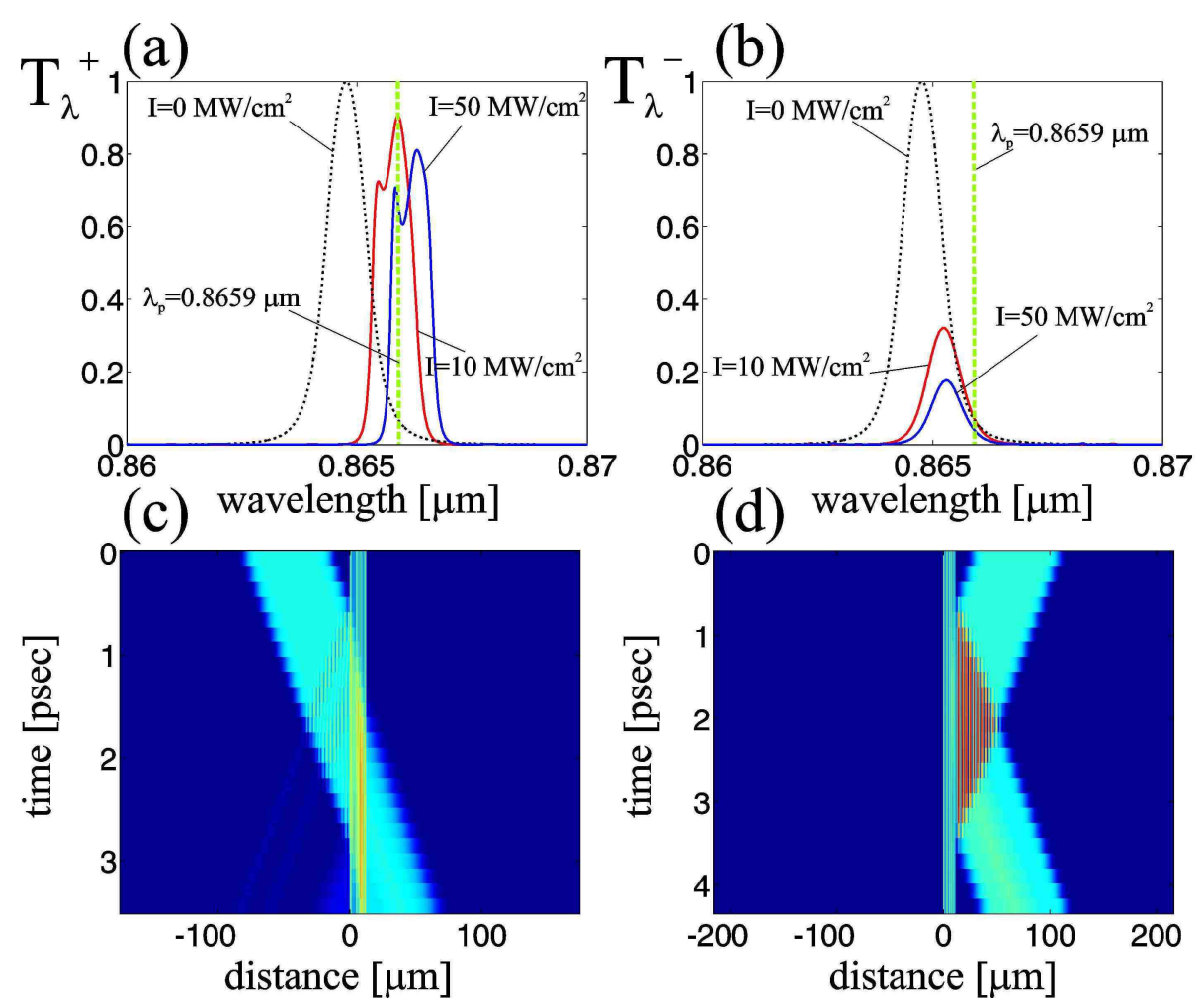

Figure 5: Color online) (a,b) Sketch of nonlinear shifts for forward [(a)] and backward [(b)] incidence, for three different input intensities. (c,d) Results of pulsed simulations, showing the asymmetric transmission properties of the nonlinear device for forward [(c)] and backward [(d)] incidence. In both cases, input intensity is $I=10 \mathrm{MW} / \mathrm{cm}^{2}$. Input pulse has a supergaussian profile, $A(\xi, \tau=0)=\sqrt{I} \exp \left[-\left(\xi / \xi_{p}\right)^{m}\right]$, with $m=40$ and $\xi_{0}=90 \mu \mathrm{m}$. The crystal is represented by the vertical bar at the center of the spatial window.

refractive indices for $\mathrm{TiO}_{2}$ and 9-BCMU used in our calculations are roughly estimated to be $n_{2}\left(\mathrm{TiO}_{2}\right) \simeq 10^{-14} \mathrm{~cm}^{2} / \mathrm{W}$ and $n_{2}(9-\mathrm{BCMU}) \simeq 2.5 \cdot 10^{-11}$ $\mathrm{cm}^{2} / \mathrm{W}$. These are measured values [21, 22, obtained by using degenerate and non-degenerate pump-probe experiments. Results published in [22] show that linear and nonlinear absorptions can be minimized considerably in 9BCMU inside certain spectral regions, which are therefore suitable for device operation. Thus here we neglect these two absorptions for simplicity. The relaxation time $\tau_{\text {rel }}$ of 9 -BCMU is estimated to be around $1.6 \mathrm{psec}$. The switching time of the device is fast and equal to $\max \left\{\tau_{p}, \tau_{\text {rel }}\right\}$.

Let us take, for instance, an input pump intensity equal to $I=I_{p}=10$ $\mathrm{MW} / \mathrm{cm}^{2}$, corresponding to the curves displayed with red solid lines in Figs. $5(\mathrm{a}, \mathrm{b})$. First of all, note the strong difference in transmission between forward 
and backward incidence. This is symptomatic of a very different bistable behavior for forward and backward propagations, see also [9]. Moreover, there is a reduction of the transmission peaks with increasing intensity. By tuning the pump wavelength near the maximum of the forward incidence peak $\left[\lambda_{p} \simeq 0.8659 \mu \mathrm{m}\right.$, see green vertical dashed line in Fig. [5(a)], one has substantial transmission for forward incidence of pump light $\left(T_{\lambda_{p}}^{+}=0.905\right)$. However, for the same wavelength, the backward transmission is located on the tail [see green vertical dashed line in Fig. 5(b)], which ensures a very low transmission for the backward direction $\left(T_{\lambda_{p}}^{-}=0.06\right)$. For the particular case shown in Figs. [5(a,b), this gives an efficient diode action with an AOD contrast equal to $C=0.8756$. The resonant nature of the PR enhances the nonlinear effects to a point that the AOD action is obtained by using modest intensities.

Finally, in order to confirm the results obtained above for realistic quasiCW pulses, which have a small but finite spectral width well inside the resonance, we accurately solve Maxwell's equations in presence of an inhomogeneous permittivity $\epsilon(z)$, which models the quasiperiodic crystal along the propagation direction $z$, normal to the layers. In order to do this, we write first of all the 1D Maxwell equations in the conventional form $\epsilon(z) \partial_{t} E+c \partial_{z} B=0, \partial_{t} B+c \partial_{z} E=0$, where $E(z, t)$ and $B(z, t)$ are the scalar electric and magnetic fields respectively, $c$ is the speed of light in vacuum. $\epsilon(z)^{1 / 2}=n(z)+n_{2}(z)|E|^{2}$ is the dielectric permittivity, where $n(z)$ is the refractive index (real and $\geq 1$ ), $n_{2}(z)$ is the Kerr-nonlinear refractive index, both of which are step functions of $z$. $I(z, t)$ is the electric field intensity. We then decouple the fast spatiotemporal oscillations due to the carrier wavenumber $k_{0}$ and frequency $\omega_{0}$ with the expansion: $E(z, t)=\left[A(z, t) e^{i k_{0} z-i \omega_{0} t}+\right.$ c.c. $] / 2, B(z, t)=\left[G(z, t) e^{i k_{0} z-i \omega_{0} t}+\right.$ c.c. $] / 2 . A$ and $G$ are the complex envelopes of the electric and magnetic fields respectively, and $k_{0}=\omega_{0} / c$. After defining new dimensionless variables $\xi \equiv z / z_{0}$ and $\tau \equiv t / t_{0}$, where $z_{0}$ and $t_{0}$ are respectively arbitrary spatial and temporal scales, we choose $z_{0}=c t_{0}$ and $t_{0}=2 \pi / \omega_{0}$. This leads to the dimensionless equations

$$
\begin{array}{r}
\partial_{\tau} A+\frac{1}{\epsilon} \partial_{\xi} G+2 \pi i(G / \epsilon-A)=0 \\
\partial_{\tau} G+\partial_{\xi} A+2 \pi i(A-G)=0
\end{array}
$$

which are suitable for numerical computations, and do not rely on any slowvarying amplitude approximation [15]. Eqs.(1)2) are solved by first initializing $A$ as a function of $\xi$, at $\tau=0$, and then posing $G(\xi, \tau=0)= \pm n(\xi) A(\xi, \tau=$ $0)$, where the plus (minus) sign refers to a forward (backward) propagation 
condition [15, which is used to test the forward/backward asymmetry in our Thue-Morse structure.

Results of simulations are shown in Fig. 5(c,d). Fig. 5(c) shows the nonlinear transmission of a forward supergaussian pulse with a spatial width $\xi_{p}=90 \mu \mathrm{m}$ ( $\gg$ than the structure's length, which is $12.09 \mu \mathrm{m}$ ), and input wavelength $\lambda_{p}=0.8659 \mu \mathrm{m}$, corresponding to the location of the green dashed line in Figs. 5(a,b). The chosen supergaussian profile well approximates the incidence of a finite $\mathrm{CW}$ wave, and input intensity was taken to be $I=10 \mathrm{MW} / \mathrm{cm}^{2}$. According to the previous discussions, this allows almost unitary transmission $\left(T^{+} \approx 0.9\right)$ if the spectral width of the pulse is narrower than that of the PR. Launching the same pulse in the backward direction we have a dramatically different behavior: Fig. 5(d) shows the nonlinear transmission in this case, where light is almost entirely reflected $\left(T^{-} \approx 0.05\right)$, confirming both qualitatively and quantitatively the optical diode action and the plane-wave predictions discussed above.

Our novel design represents an excellent compromise between contrast, total size, intensity requirements and simplicity. For instance, some of the previous approaches show nearly unitary contrast, good intensity requirements but large size [4, 5]; while other devices show cumbersome design [7, 6, 8].

In conclusion, we have studied numerically the linear and nonlinear transmission properties of Thue-Morse quasiperiodic PCs near a PR, i.e. a sharp resonance located in the pseudobandgap, which behaves very much like a defect state with a relatively high Q-factor, and is due to the action of AndersonKohmoto localization in the crystal. We have shown that the strong asymmetry of odd-order Thue-Morse lattices, combined with a Kerr nonlinearity, gives rise to a highly nonreciprocal transmission which is the major feature of an AOD, and this effect has also been confirmed by numerically integrating Maxwell's equations for realistic optical pulses. Serious advantages of our design are small size (of the order of a few microns) and low operational optical intensity, a consequence of the intrinsic antisymmetry of the structures considered and the resonant nature of the transmission.

This work is supported by the UK Engineering and Physical Sciences Research Council (EPSRC).

\section{References}

[1] E. Yablonovitch, Phys. Rev. Lett. 58, 2059 (1987); S. John, Phys. Rev. Lett. 58, 2486 (1987). 
[2] P. Yeh, Optical Waves in Layered Media (John Wiley and sons, Inc. Hoboken, New Jersey, 2005).

[3] M.Scalora et al., J. Appl. Phys. 76, 2023 (1994); Tocci et al., Appl. Phys. Lett. 66, 2324 (1995).

[4] K. Gallo and G. Assanto, Opt. Lett. 16, 267 (1999).

[5] K. Gallo et al., Appl. Phys. Lett. 79, 314 (2001).

[6] S. Mujumdar and H. Ramachandran, Opt. Lett. 26, 929 (2001).

[7] S. Pereira et al., Photonics and Nanostructures - Fundamentals and Applications 2, 181 (2004).

[8] M. H. Song et al., Thin Solid Films 509, 49 (2006).

[9] M.W. Feise, I. V. Shadrivov and Y. S. Kivshar, Phys. Rev. E 71, 037602 (2005).

[10] E. L. Albuquerque and M. G. Cottam, Polaritons in Periodic and Quasiperiodic Structures (Elsevier, Amsterdam, 2004).

[11] Nian-hua Liu, Phys. Rev. B 55, 3543 (1997).

[12] D. Schechtman et al., Phys. Rev. Lett. 53, 1951 (1984).

[13] R. Merlin et al., Phys. Rev. Lett. 55, 1768 (1985); Z. Cheng, R.Savit and R. Merlin, Phys. Rev. B 37, 4375 (1988).

[14] A. Thue, Norske Vididensk. Selsk. Skr. I. 7, 1 (1906).

[15] F. Biancalana et al., Phys. Rev. E 75, 046607 (2007).

[16] D. Ripin et al., IEEE J. Light. Tech. 17, 2152 (1999).

[17] X. Q. Huang et al., Phys. Rev. B 63, 245104 (2001).

[18] F. Qiu et al., Europhys. Lett. 68, 658 (2004).

[19] J. Hwang et al., Opt. Lett. 31, 377 (2006).

[20] P. W. Anderson, Phys. Rev. 109, 1492 (1958); W. Gellermann, M. Kohmoto, B. Sutherland and P. C. Taylor, Phys. Rev. Lett. 72, 633 (1994); H. Hiramoto and M. Kohmoto, Phys. Rev. Lett. 62, 2714 (1989).

[21] R. Adair, L. L. Chase and S. A. Payne, Phys. Rev. B 39, 3337 (1989). 
[22] S. Molyneux et al., Opt. Lett. 18, 2093 (1993); A. K. Kar, Polym. Adv. Technol. 11, 553 (2000). 